\title{
On the Collection and Use of Fish Scales for Fisheries Management: Pros, Cons, and Potential Solutions
}

\author{
Patrick Wahlig $^{1}$ and Nathalie Sommer ${ }^{\#}$ \\ ${ }^{1}$ Falmouth High School, Falmouth, MA, USA \\ \#Advisor
}

ABSTRACT

Fisheries have enormous ecological and economic importance to the planet, yet threats of overfishing and climate change are altering the structure of the world's fisheries. In order to assess and properly manage fisheries, information including fish age, size, growth rate, and origin must be gathered. Various fish body structures can yield this data but acquiring such information requires lethal sampling and arduous analysis techniques. Fish scales, however, do not present such limitations, as scales are ubiquitous, can be sampled nonlethally, and are inexpensive to analyze. Fish scales have known drawbacks which have stymied full adoption by fisheries management. Current issues with scales are discussed, specifically scale resorption and regeneration, and practical solutions to these issues are proposed. While scales have a long history of utility in fisheries management, their potential for wider application remains enormous. The synthesized information and suggested studies in this review offer a path forward for scale analysis to become more efficient and accurate, thereby eliminating lethal sampling methods while improving fisheries management.

\section{Introduction}

Sustainable fisheries management is critical in supporting both the planet's economy and ecosystems. Fisheries provide massive economic support to countries around the globe, supporting an estimated 260 million jobs worldwide in 2011 (Teh \& Sumaila, 2011). In the United States, the fishing industry alone contributed 100 billion dollars to the Gross Domestic Product (NOAA, 2020). Perhaps more important than the face-value economic support fisheries offer is the fact that organisms comprising fisheries are the stabilizing consumers of nearly every aquatic food web (NOAA, 2019). Fish are biochemical keystones, providing a critical mass of nutrients necessary for primary producers to grow and develop (Allgeier et al., 2013). Their overall function as nutrient recyclers means fish have an outsized effect on their ecosystem (McIntyre et al., 2007). A loss of diversity in fisheries would therefore have a radical effect on ecosystem productivity, putting entire marine food webs in peril (McIntyre et al., 2007).

Overfishing places coastal ecosystems under severe stress. When species are overfished, their population levels can be driven so low that they no longer interact significantly with other species in their community, resulting in functional extinction (Jackson et al., 2001). When commercial and recreational fisheries target the large fish at the top of the marine food chain, it leads to a phenomenon known as mesopredator release, in which smaller predatory fish populations (the prey of the fish targeted by fisheries) rapidly rise in density, straining existing coastal habitats and reducing water quality (Eriksson et al., 2011). While overfishing is mainly discussed in reference to marine and coastal ecosystems, inland fisheries also experience overfishing, with a consequent decline of freshwater biodiversity (Allan et al., 2005).

In order to mitigate the threat of overfishing, proper fisheries management is needed. Fisheries management sets fishing regulations and quotas, aiming to protect and sustain marine ecosystems (NOAA Fisheries). Through the use of stock assessments and other scientific observations, fisheries management organizations such as the National Oceanic and Atmospheric Administration seek to balance stock harvest and stock size, striving to create a sustainable harvest of global fish populations (NOAA Fisheries). 


\section{Analysis of Fish Hard Parts for Fisheries Management}

In order to properly manage any fish stock, fisheries management organizations must take into account variables such as fish age, size, and growth patterns (Klein et al., 2017). Data on fish age is crucial in formulating catch-at-age assessments, which can then be used to measure fish population levels and mortality rates, as well as age-based fishery selectivity (Linton \& Bence, 2010). Setting sustainable guidelines also requires an understanding of the speciesspecific rate and pattern of growth (Khan \& Khan, 2014). Especially when dealing with migratory fish, knowledge of fish natal origins, or where the fish are currently being recruited (born and developed), is necessary to prescribe regional protection for especially productive areas. Without such protection, fish populations would crash due to lack of adequate recruitment. Furthermore, as the threat from climate change and warming waters continues to grow, knowledge of fish origin could be used to examine the impacts of climate change on the complex interactions among species of any aquatic ecosystem. Available evidence suggests that climate-influenced change in fish recruitment is the driving force behind changes in fish population structure (Rijnsdorp et al., 2009). Understanding fish natal origins (alongside data on age and growth patterns) could be the key to tracking climate-induced shifts in recruitment and the long-term implications of climate change on our marine world. Especially important is the use of a nonlethal acquisition method in fisheries science, as it will provide researchers with "cheaper", more readily accessible data without further compromising the population growth of overfished species.

Various body structures on fish have the potential to yield such data, including scales, vertebrae, sagittae, and fin spines (Maraldo \& MacCrimmon, 1979; Welch et al., 1993; Murie et al., 2009; Figure 1). Some of these structures offer the advantage of non-lethal sampling. Lethal data collection methods are often burdensome due to the difficulty of extraction and collection. Furthermore, when dealing with an overfished species, restrictive fishing regulations and the potential for negative public perception make it extremely difficult to collect representative stock data using lethal data collection methods (Klein et al., 2017). Consequently, data acquired through nonlethal means is preferred in many situations. 


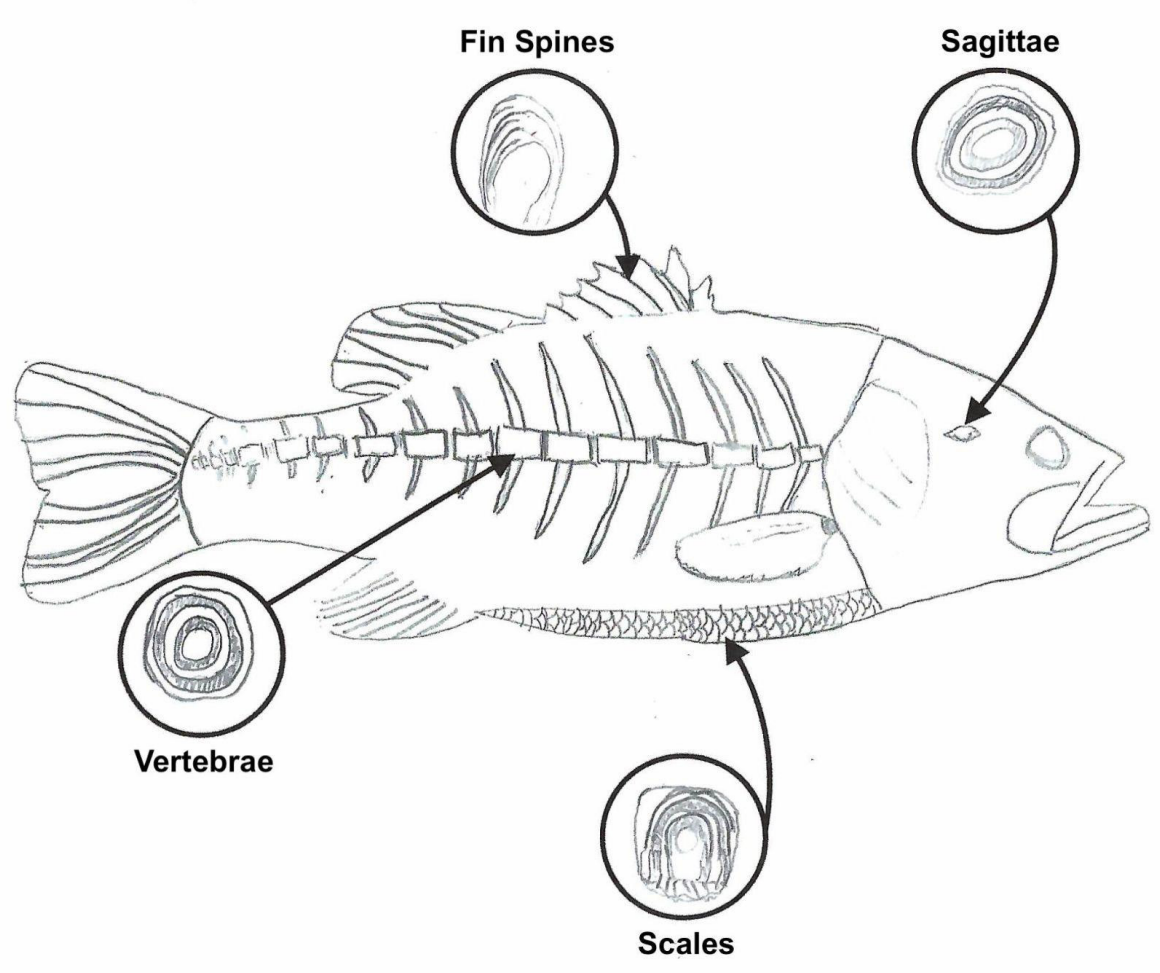

Figure 1. Bodily locations of fish hard parts used in fisheries management. Fish hard parts can be collected and interpreted for stock-relevant life history metrics. While sagittae are often regarded as the "gold standard" for body structure for reliable life history metrics, scales and spines are nonlethal alternatives to sagittae in data collection. Vertebrae are often analyzed in Elasmobranchii, as that subclass of fish lack sagittae and their scales do not grow continuously (MarAlliance, 2018). To sample vertebrae, the organism must be euthanized.

Structures on fish that provide representative age and growth information can also contain valuable elemental information regarding fish natal origin, and therefore be used to profile the recruitment zones for migratory species. As these structures contain indicators representative of the body of water a fish inhabits at a given time in its growth cycle, the structural component corresponding to the earliest portion of the fish's life could be analyzed to determine natal origin (Kerr \& Campana, 2014). The efficacy of scales and spines in origin determination can, however, be negatively affected by periods of nutritional scarcity. Fish will actually resorb nutrients present in these structures if their diet is compromised (Kerr \& Campana, 2014).

The vertebrae of the Elasmobranchii family (i.e. skates, sharks, and rays) form yearly bands of growth. In these vertebrae, paired translucent and opaque bands (annuli) represent one year of growth (Kerr \& Campana, 2014). As vertebrae begin to form in the earliest stages of a fish's life, these annuli can be used to determine the age of the fish and measure fish growth, similar to counting the rings on the cross-section of a tree. Vertebrae, however, are a lethal form of data collection, as they must be removed from the spine of the fish.

The sagittae, one of the ear bones (otoliths) of bony fish, grow in a similar way and can also be used to estimate the age of fish. Similar in concept to vertebrae, a pair of one translucent and one opaque band represents one 
year of growth in sagittae (NOAA Fisheries). As otoliths are taken from the brain cavity of sampled fish, otolith extraction is, like vertebrae harvest, a lethal technique (Klein et al., 2017).

Fish fin spines and rays also demonstrate formation of growth bands, and are therefore a suitable structure for age determination (Campana et al., 2006). There can be substantial error in age estimates, however, due to normal wear and tear on a fish's spines which erodes the growth bands. Fin spines, therefore, might not provide an accurate age profile of the fish. (Campana et al., 2006). The sampling of spines and rays, while nonlethal, is more invasive than that of scales, which is made evident when comparing the sampling procedures involved. The sampling of fish spines involves the potentially injurious clipping or amputating of external structures that are firmly embedded in the substance of the fish.

Scale acquisition is a comparatively atraumatic process, where scales can be gently scraped or tweezed away without any gross anatomic or functional disruption of the organism. Scales are numerous, superficial, and acquired with ease. Scale sampling does not severely disrupt the structural integrity of the organism and can be removed by simply pulling them from the dermis pocket.

Assessment of growth increments through the use of scales is relatively straightforward, as individual "circuli" (rings) form clear, readily-identifiable annuli, which represent one year of growth. These annuli are then counted to determine fish age (Garling, 2016). Although scales are an attractive, non-lethal data source, problems have been reported with scale loss, regeneration, and inaccurate age measurements in older fish (Kerr \& Campana, 2014). In many species, scales can underrepresent the age of older fish that have begun to senesce (Lowerre-Barbieri et al., 1994; Maceina \& Sammons, 2006). This potential to underestimate fish age must be factored into stock management and when modeling age-based fishery selectivity. In selected species, older fish are more likely to breed. If their age is consistently underestimated, inadvertent and undesirable harvest of this critical fish demographic could trigger population collapse. (Horká et al., 2010).

Other issues related to scale wear (e.g. shedding and regeneration) can be combated through multi-site sampling, as there are a variety of potential sampling regions available on the fish body. Locations of frequent scale turnover and increased scale regeneration on the fish body should be established and avoided in order to increase project efficiency and accuracy (Abdu-Nabi, 1983).

While both scales and fin spines show promise as nonlethal alternatives to sagittae and vertebrae in origin determination and aging estimates, scales have the clear advantage of being ubiquitous on the fish body. If fish spines are worn down, it is extremely difficult to establish an accurate age profile of that particular fish (Kerr \& Campana, 2014). This is not an issue with scales, however, as scales from a more preserved region on the fish body could readily be sampled if another site was damaged or worn.

Scales are easy to sample and inexpensive to analyze as compared to other structural sources, such as sagittae and fin spines, which require a time-consuming preparation process involving advanced equipment (Davies et al., 2017; Davies et al., 2015; Koch \& Quist, 2011). Use of scales leads to a larger possible sample size, as more samples can be analyzed in the same amount of time, and at less cost. Scales can also be sampled from an individual fish multiple times throughout its life. Repeatedly-sampled fish could provide longitudinal project data, making it possible to conduct growth studies that require the recapture of a sampled fish. Critically, removing the scales from a fish does not require euthanasia, encouraging the preservation and rehabilitation of an overfished or threatened species.

\section{Background on Fish Scales}

The presence of scales on fish is mainly for protection. In fact, early forms of extant fish had scales of such size and multitude that, while they provided protection, they hindered the ability for the fish to move efficiently (Burdak, 1986). As fish evolved, the reduction in the integumental skeleton (the "outside" of the fish body) led to scales taking on different forms in order for the fish to achieve efficient locomotion.

There are four types of fish scales, each with their own distinct shape. Those scale groups are cycloid, ctenoid, ganoid, and placoid (Rawat, 2021; Figure 2). Placoid scales are spiny projections characteristic of cartilaginous fish 
like sharks. Ganoid scales are similar to placoid scales and are usually rhomboid in shape. Cycloid scales are oval in shape and are found in carp-like fish species. Lastly, ctenoid scales are similar to cycloid scales, the main difference being that ctenoid scales have spines along their free edges ("Integument", 2011). In teleosts (which comprise more than $99.8 \%$ of ray-finned fishes), scales are either cycloid or ctenoid. These scales are thin, overlap on the fish body, and are mineralized (Kawasaki, 2016; Volff, 2004). In some species, such as the goldfish Carassius auratus, scales contain about $20 \%$ of total body calcium, suggesting that scales may serve as a type of nutrient reservoir for the fish (Tagaki et al., 1989).

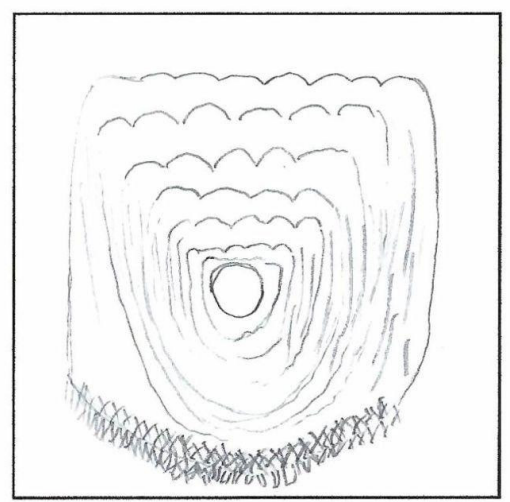

Ctenoid Scale

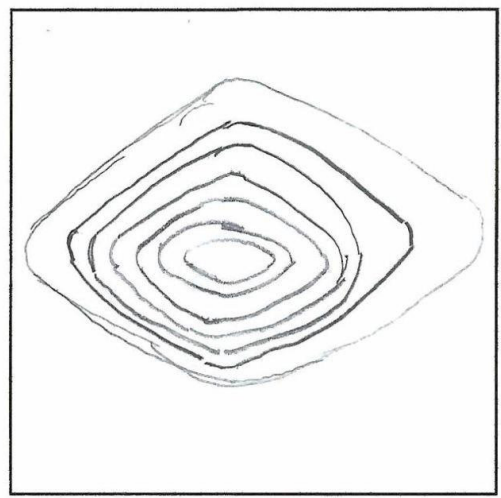

Ganoid Scale

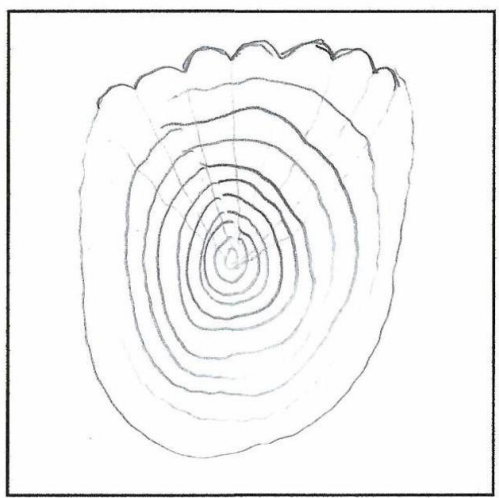

Cycloid Scale

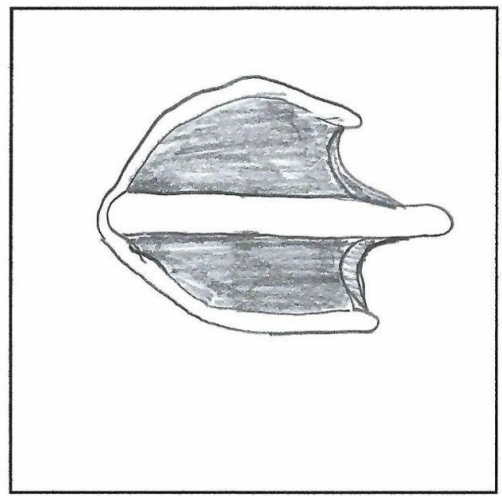

Placoid Scale

Figure 2. The four types of fish scales. Ctenoid scales are often found on spiny-rayed fishes: they can be identified by the spines along the base of the scale. Cycloid scales are more common in soft-rayed fishes and are similar in form to ctenoid scales, the difference being that cycloid scales lack spines along their base. Ganoid scales are hard, bony scales found in species like the sturgeon and gar. Finally, placoid scales are characteristic of cartilaginous fishes, with their unique toothed structure functioning to reduce hydrodynamic drag.

\section{Current Problems with Fish Scales}

\section{Scale Regeneration}


While scales are heavily involved in fish protection and appear to be used in nutrient storage, they are shed quite often by almost all teleost fish. Some species shed them in conjunction with major life events, as upon reaching sexual maturity and settling into their permanent habitat. (Bereiter-Hahn \& Zylberberg, 1993). Lost scales are rapidly replaced by regenerated ones, restoring the protective integumentary layer of the fish. The number of regenerated scales tends to increase with age in all species, as original scales are either shed, worn down, or are removed by injury (Bereiter-Hahn \& Zylberberg, 1993).

Regenerated scales are rapidly grown and mineralized, and thus do not share the same form as original scales (Figure 3). In teleost fish, original scales exhibit annuli, which can be used to age the fish (Garling, 2016). This is generally not the case in regenerated fish scales. Regrown quickly, these scales often do not exhibit clear annuli and do not reflect the fish's entire life span (Davies et al., 2015; Figure 3). Furthermore, in a migratory species such as the striped bass, there is a high likelihood that a regenerated fish scale would not incorporate the biochemical profile of the body of water in which the fish was born, rendering it inaccurate for origin analysis. As fish origins can be determined through chemical analysis of a selected structure, a sample representative of the fish's natal environment is needed (Kerr et al., 2019; Avigliano et al., 2017).
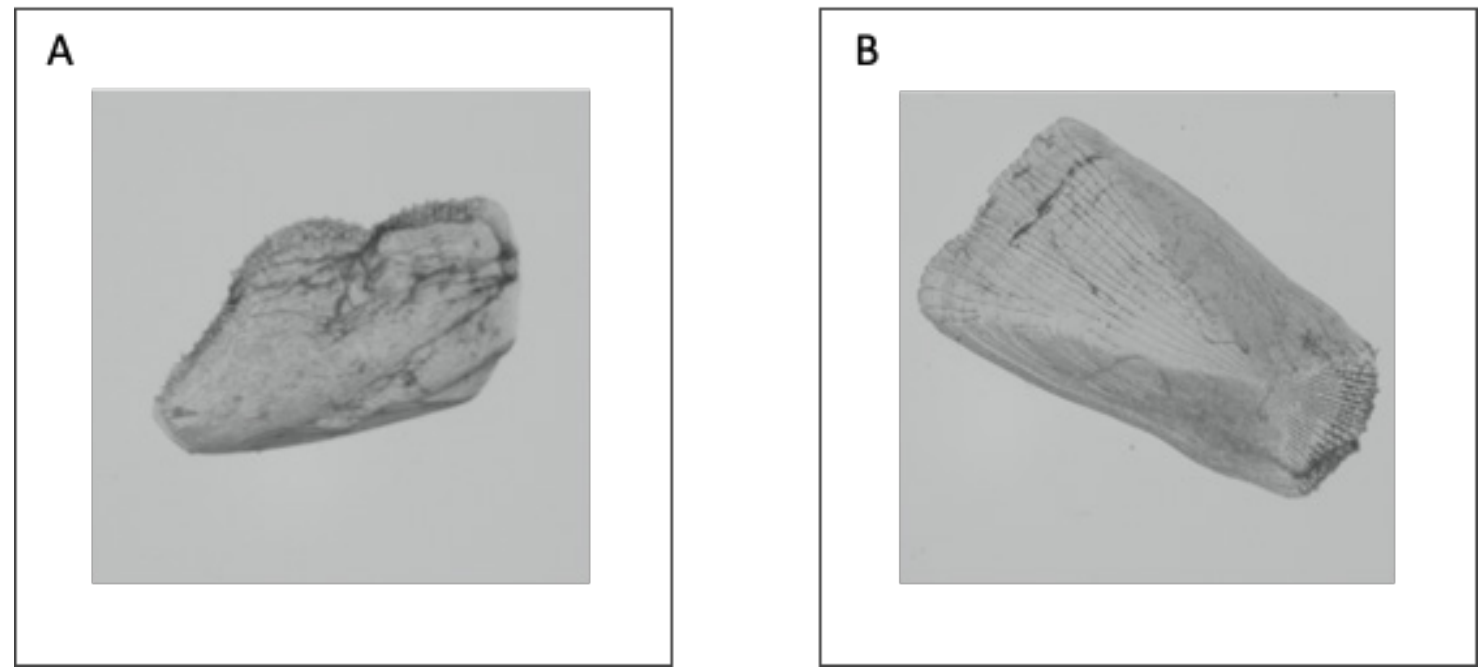

Figure 3. Regenerated striped bass scale versus original striped bass scale. Part A represents a regenerated striped bass scale: its radii lack a "V" shape, clear focus is not present. Part B represents an original striped bass scale: its radii form a "V" shape and a clear focus (center).

In any study that aims to obtain age or origin information from fish scales, regenerated scales pose a serious obstacle. They contribute no representative data, are time-consuming to analyze for a large number of individuals, and contribute to project expenses. In one study conducted by Hellmair et al. (2019) on juvenile Oncorhynchus mykiss in the Salinas River Basin, it was noted that roughly 20 percent of all samples could not be analyzed due to scale regeneration or lack of clear annuli (Hellmair et al., 2019). A 20 percent decrease in data can compromise the validity of a study and impede the compilation of representative results. In grant-funded research, financial resources are finite. If time and money is wasted analyzing regenerated scales, it is likely that a project's goals will not be met, nor will representative results be attained.

\section{Scale Resorption}

Some species exhibit scale resorption, a process in which scales are absorbed into the fish's body. Resorption can occur during times of fasting, for instance when salmon travel upstream to spawn (Kacem et al., 2013). Scales are 
resorbed as a means of gathering nutrients that may not be available to the fish at a particular time. In the case of the Atlantic salmon, resorbed scales serve to supplement the fish's supply of minerals used during reproduction. Despite salvaging nutrients from resorbed scales, many salmon are so nutrient-deprived that they die soon after reproduction. These fish completely expend their energy in the process of swimming upstream and spawning (Halttunen, 2011). Resorbed portions of scales appear clear and lack circuli, confounding age and growth rate determination. Resorption also affects the biochemical profile of the scale, making determination of origin impossible (Love, 2016; Rijnsdorp et al., 2009). Reassuringly, unless a species is known to go through long-term periods of nutrient scarcity as in the case of the Atlantic salmon, it is unlikely that resorption will occur to such a degree that there is no means of obtaining stock-relevant information ("A Basic Guide to Aging \& Identification of Pacific Salmon Scales"; Day et al.). While there is no solution to scale resorption, conducting analyses of rates of resorption of scales across a fish's body will determine which areas provide the greatest density of unabsorbed scales.

\section{Solutions to Regenerated Scales}

As fish will regenerate lost scales in locations of injury, identifying sites of frequent injury and scale shedding will help researchers lower the frequency of sampling non-representative, regenerated scales. Identifying such hot spots will need to be species-specific and must take into consideration the functional community dynamics at play. Generally, it is recommended that scales be selected from the body region where they first form, as they will contribute the most accurate age data (Longo et al., 2020). In some fast-growing species, however, the risk of obtaining a regenerated scale discourages sampling from an "old" scale region on the fish body. For instance, it has been observed in the striped bass that scales nearest to the lateral line form earliest, but these scales have only an approximate 30day head start when compared to scales from other candidate sampling sites (Galbraith et al.). Scales are aged by seasons, not days. Therefore, it may be more efficient to focus on avoiding regenerated scales, as opposed to selecting scales from regions that form earliest, given that formation times are relatively similar across the fish body.

An example of the importance of understanding fish scale regeneration when evaluating fish age can be found when fish eyespots are examined. There is evidence that piscine predators hunt by honing in on the eyes of their prey (Kjernsmo \& Merilaita, 2013). Fish with noticeable or larger eyes would logically be more commonly attacked at their head. Conversely, fish with eyespots ("fake" eyes designed to deter predators from vital organs) might have a higher rate of injury - and consequently a higher rate of regeneration - in areas where eyespots are present. Additionally, abiotic loss of scales related to fish habits such as rock scraping, bottom feeding, or hole burrowing, must also be taken into account. These mechanisms of scale loss are a consequence of body size and swimming habits in different environments.

Common locations of scale loss must be investigated in individual fish species before extensive scale analysis is used in fisheries management, in order to avoid a high occurrence of regenerated scales. In order to determine areas of high regeneration on fish bodies, a study could be conducted analyzing photos of caught fish to visually identify sites of injury. Scales could be sampled from injured fish to compare the occurrence of regenerated scales in the injury site with scales from healthy fish used as a control variable. This would be most successful through the use of citizen science, with anglers submitting pictures of their caught fish to a central organization for analysis, similar in process to the Snap-a-Striper project conducted by the Gulf of Maine Research Institute, where fishermen are encouraged to submit photos of their catch next to a standardized data card for morphometric analysis. While the goals of the proposed study would be different from those of the Snap-a-Striper program, the concept of citizen science and photo collection could still be utilized effectively ("Snap-a-Striper."). Photos of fish deemed healthy and injury-free by researchers could be compared to submitted photos, and a machine-learning algorithm might be used to discriminate pictures of injured fish from pictures of healthy fish, increasing the efficiency of such a research project.

There may be other factors at play that might not be discernible from a simple visual inspection. Because of this, an in-depth analysis of a fish's scale profile across its body is ideal, similar to the one conducted by Abdu-Nabi (1983) on yellow perch. Scale regeneration across multiple body areas was considered a key factor when selecting 
fish scales for growth studies of yellow perch (Abdu-Nabi, 1983). Alternatively, an efficient analysis of 3-5 main body regions on a fish could be conducted, simply to determine the lowest average rates of scale regeneration. A number of scales could be collected from each area, and the overall percentage of regrowth versus original scales could be calculated. An analysis of variance could then be conducted to discern statistically significant differences between sampling sites. The location that has the lowest chance of yielding a regenerated scale could be ascertained for any given fish species following the methods described above. Such an exercise might improve the merits of fish scales as an effective nonlethal data source for fisheries management.

\section{Conclusion}

Fisheries are of crucial economic and ecological importance worldwide. Fisheries management organizations are vital for consistent protection of these important natural resources. In order to properly manage fisheries, information relating to fish demographics must be discerned.

There are several structures that have shown utility in the acquisition of representative age, growth, and origin data. Certain structures (such as sagittae and vertebrae) require euthanasia of the fish, which is an unattractive feature, especially when dealing with threatened fish species and public perception (Klein et al., 2017). Other structures, such as fin rays and spines, can be sampled non-lethally. These structures, however, have displayed variability in age determination due to growth increment destruction and wear of spines in some species (Kerr \& Campana, 2014). Alternatively, scale sampling provides the highest availability of data, as scales are nearly ubiquitous on fish bodies, with the exception of a few fisheries-peripheral species such as the ocean sunfish and the naked catfish (Ramel, 2020). Fish scale acquisition is also non-lethal. Fish scales are easy to collect, prepare, and analyze, making them the preferred aging structure for many species (Davies et al., 2015).

Often associated with fish scales is the issue of regeneration. When fish scales are shed or removed from a fish, a regenerated scale forms, taking the place of the original scale. These regenerated scales do not reflect a fish's entire life span, limiting their use in stock assessments, especially in regard to migratory fish origin (Davies et al., 2015; Ericksen, 1999). In order to enhance the use of scales as a data source, the issue of scale regeneration must be anticipated and countered. As scale loss varies between fish species, it is not enough to assume inter-species similarities in locations of scale regeneration. Thus, it is recommended that studies conduct research outlining "hot spots" of scale regeneration on the species to be analyzed in order to make the most of a study's time, money, and effort. In some species, scale resorption is also problematic. This phenomenon seems to occur only in fish that undergo long periods of nutrient scarcity, but must be taken into account if the species is known to be subjected to such an ordeal (Kacem et al., 2013).

Scales have a long and diverse history in the world of fisheries management, believed to have first been analyzed by Aristotle in 340 B.C. to determine fish age (MarAlliance, 2018). Scales have great potential for further research applications, whether that be as tools for aging, origin identification, or the monitoring of aquatic ecosystems (Laine et al., 2011; Pouilly et al., 2014; Trueman \& Moore, 2007).

The use of scales to determine fish origin could prove critical in shaping our understanding of the effects of climate change on our world and its oceans. The ability to trace the natal origins of migratory fish could be used to assess the impact of climate change on the complex interactions among organisms within any large body of water, such as the Atlantic Ocean. As evidence suggests that climate-influenced changes in recruitment are the driving force behind changes in fish populations, understanding how fish origins are changing in response to climate change is vital (Rijnsdorp et al., 2009). Conveniently and perhaps ironically, the very organisms affected so harshly by climate change could contain the answers to the challenges global warming poses today. 


\section{Acknowledgments}

I would like to thank my mentor Nathalie Sommer for guiding me in this project.

\section{References}

Abdu-Nabi, Ahmad Hassan. "Factors Related to Selection of Fish Scales for Growth Studies of Yellow Perch, Perca Flavescens." Iowa State University Digital Repository, 1983,

https://lib.dr.iastate.edu/rtd/7693/?utm source=lib.dr.iastate.edu\%2Frtd\%2F7693\&utm medium=PDF\&utm campai $\mathrm{gn}=$ PDFCoverPages.

Allan, J. David, et al. "Overfishing of Inland Waters." OUP Academic, Oxford University Press, 1 Dec. 2005, https://academic.oup.com/bioscience/article/55/12/1041/407055?login=true.

Allgeier, Jacob E., et al. "Consumers Regulate Nutrient Limitation Regimes and Primary Production in Seagrass Ecosystems.” The Ecological Society of America, John Wiley \& Sons, Ltd, 1 Feb. 2013, https:/esajournals.onlinelibrary.wiley.com/doi/abs/10.1890/12-1122.1.

Avigliano, Esteban, et al. "Otolith Elemental Fingerprint and Scale and Otolith Morphometry in Prochilodus Lineatus Provide Identification of Natal Nurseries." Fisheries Research, Elsevier, Feb. 2017, https://www.sciencedirect.com/science/article/abs/pii/S0165783616302387?via\%3Dihub.

"A Basic Guide to Aging \& Identification of Pacific Salmon Scales.” Alaska Department of Fish \& Game, https://www.adfg.alaska.gov/static/education/educators/curricula/pdfs/salmon scales guide aging identification.pd f.

Bereiter-Hahn, J, and L Zylberberg. "Regeneration of Teleost Fish Scale." Comparative Biochemistry and Physiology Part A: Physiology, Pergamon, Aug. 1993, https://www.sciencedirect.com/science/article/abs/pii/0300962993902623.

Burdak, V D, et al. "Morphologie Fonctionnelle Du tégument écailleux Des Poissons." Find in a Library with WorldCat, 1986, https://www.worldcat.org/title/morphologie-fonctionnelle-du-tegument-ecailleux-despoissons/oclc/18805405.

Campana, Steven E., et al. "Bomb Dating and Age Validation Using the Spines of Spiny Dogfish (Squalus Acanthias)." Environmental Biology of Fishes, Kluwer Academic Publishers, 12 Oct. 2006, https://link.springer.com/article/10.1007/s10641-006-9107-3.

Davies, James, et al. Protocol Age Estimation of Otolith Transverse Cross-Sections for Striped Bass Morone Saxatilis. Center for Quantitative Fisheries Ecology, 2017, https://ww1.odu.edu/content/dam/odu/offices/center-forquantitative-fisheries/docs/striped-bass-otolith-ageing-protocol.pdf.

Davies, James, et al. Protocol Preparation of Otolith Transverse Thin-Sections for Age Estimation of Bluefish Pomatomus Saltatrix. Center for Quantitative Fisheries Ecology, 2015, https://ww1.odu.edu/content/dam/odu/offices/center-for-quantitative-fisheries/docs/striped-otolith-preparationprotocol.pdf. 
Davies, James, et al. Protocol Preparation of Scales for Age Estimation of Striped Bass Morone Saxatilis. Center for Quantitative Fisheries Ecology, 7 Oct. 2015, https://www.odu.edu/content/dam/odu/offices/center-for-quantitativefisheries/docs/STRIPED\%20BASS\%20SCALE\%20PREPARATION\%20LATEX\%20MAIN\%20DOCUMENT.pdf

Day, Albert M., et al. "Fishery Bulletin of the Fish and Wildlife Service, Volume 50.” Google Books, Google, https://books.google.com/books?id=7ugtBjlU1vMC\&pg=PA20\&lpg=PA20\&dq=scale $\% 2$ Bresorption $\% 2 B$ in $\% 2 B s t r$ iped $\% 2$ Bbass \&source $=$ bl\&ots=TG5-EKo8Wt\&sig $=$ ACfU3U3q6hJkAH-

XANwaTsUd3XqoPaqxIw\&hl=en\&sa=X\&ved=2ahUKEwim5o7Vs8zyAhXsmOAKHTjrAUMQ6AF6BAgiEAM\# $\underline{\mathrm{v}=\text { onepage } \& \mathrm{q}=\text { partially } \& \mathrm{f}=\text { false. }}$.

Ericksen, Randolph P. Scale Aging Manual for Coastal Cutthroat Trout from Southeast Alaska, Alaska Department of Fish and Game, Dec. 1999, http://www.adfg.alaska.gov/FedAidpdfs/Sp99-04.pdf.

Eriksson, Britas Klemens, et al. "Effects of Altered Offshore Food Webs on Coastal Ecosystems Emphasize the Need for Cross-Ecosystem Management.” Ambio, Springer Netherlands, 24 June 2011, https://link.springer.com/article/10.1007/s13280-011-0158-0.

Galbraith, John, et al. "Striped Bass Morone Saxatilis (Walbaum 1792) - Buzzards Bay." Striped Bass, Buzzards Bay National Estuary Program, https://buzzardsbay.org/wp-content/uploads/2017/03/striped-bass.pdf.

Garling, D. L. "Determining the Age of Fish (E1774).” MSU Extension, Feb. 2016, https://www.canr.msu.edu/resources/determining the age of fish e1774.

Halttunen, Elina. Staying Alive - The Survival and Importance of Atlantic Salmon Post-Spawners. The University of Troms $\varnothing$ UIT, 2011, https://munin.uit.no/bitstream/handle/10037/3536/thesis.pdf? sequence=2\&isAllowed=y.

Hellmair, Michael, et al. Age Composition, Growth, and Life History Characteristics of Juvenile Oncorhynchus Mykiss in the Salinas River Basin. FISHBIO, Feb. 2019, http://salinasrivermanagementprogram.org/documents/Arroyo Seco Scale Report final.pdf.

Horká, P., et al. "Validation of Scale-Age Determination in European Grayling Thymallus Thymallus Using TagRecapture Analysis.” Wiley Online Library, John Wiley \& Sons, Ltd, 27 May 2010, https://onlinelibrary.wiley.com/doi/10.1111/j.1095-8649.2010.02664.x.

“Integument.” Encyclopaedia Britannica, Encyclopædia Britannica, Inc., 2011, https://www.britannica.com/science/integument/Fishes.

Jackson, Jeremy B. C., et al. "Historical Overfishing and the Recent Collapse of Coastal Ecosystems." Science, 27 July 2001, https://www.science.org/doi/10.1126/science.1059199.

Kacem, A., et al. "Resorption of Scales in Atlantic Salmon (Salmo Salar) during Its Anadromous Migration: a Quantitative Study." Société Française D'Ichtyologie - Cybium, 2013, https://doi.org/10.26028/cybium/2013-373$\underline{007}$. 
Kawasaki, Kenta C. “A Genetic Analysis of Cichlid Scale Morphology.” ScholarWorks@UMass Amherst, Sept. 2016, https://doi.org/10.7275/9004629.

Kerr, Lisa A., and Steven E. Campana. "Chemical Composition of Fish Hard Parts as a Natural Marker of Fish Stocks." Stock Identification Methods (Second Edition), Academic Press, 2014, https://www.sciencedirect.com/science/article/pii/B9780123970039000114.

Kerr, Lisa A., et al. "Mixed Stock Origin of Atlantic Bluefin Tuna in the U.S. Rod and Reel Fishery (Gulf of Maine) and Implications for Fisheries Management.” Fisheries Research, Elsevier, 13 Dec. 2019, https://doi.org/10.1016/j.fishres.2019.105461.

Khan, Salman, and Mohammad Afzal Khan. "Importance of Age and Growth Studies in Fisheries Management." ResearchGate, 2014, https://www.researchgate.net/publication/303897650 Importance of age and growth studies in fisheries manag ement.

Kjernsmo, Karin, and Sami Merilaita. "Eyespots Divert Attacks by Fish." Proceedings of the Royal Society B: Biological Sciences, 7 Sept. 2013, https://royalsocietypublishing.org/doi/10.1098/rspb.2013.1458.

Klein, Zachary B., et al. "Precision and Accuracy of Age Estimates Obtained from Anal Fin Spines, Dorsal Fin Spines, and Sagittal Otoliths for Known-Age Largemouth Bass.” BioOne Complete, Eagle Hill Institute, 2017, https://bioone.org/journals/southeastern-naturalist/volume-16/issue-2/058.016.0209/Precision-and-Accuracy-ofAge-Estimates-Obtained-from-Anal-Fin/10.1656/058.016.0209.short.

Koch, Jeff, and Michael C. Quist. "A Technique for Preparing Fin Rays and Spines for Age and Growth Analysis.” Taylor \& Francis, 2011, https://www.tandfonline.com/doi/abs/10.1577/M06-224.1.

Laine, Arnold O., et al. "Accuracy of Using Scales and Cleithra for Aging Northern Pike from an Oligotrophic Ontario Lake.” American Fisheries Society, John Wiley \& Sons, Ltd, 8 Jan. 2011, https://afspubs.onlinelibrary.wiley.com/doi/10.1577/15488675\%281991\%29011\%3C0220\%3AAOUSAC $\% 3 \mathrm{E} 2.3 . \mathrm{CO} \% 3 \mathrm{~B} 2$.

Linton, Brian C., and James R. Bence. "Catch-at-Age Assessment in the Face of Time-Varying Selectivity." OUP Academic, Oxford University Press, 24 Dec. 2010, https://academic.oup.com/icesjms/article/68/3/611/656857.

Longo, Isis, et al. A Practical Handbook for Determining the Ages of Gulf of Mexico and Atlantic Coast Fishes, Gulf States Marine Fisheries Commission, Nov. 2020, http://www.asmfc.org/files/Science/GOM AtlanticCoast FishAgeingHandbook 2020web.pdf.

Love, David C. Manual for Aging Steelhead Trout Scales Based on Scale Sampling from Sitkoh Creek, Big Ratz Creek, and Other Southeast Alaska Streams, 2003-2011. Alaska Department of Fish and Game, Dec. 2016, https://www.adfg.alaska.gov/FedAidPDFs/SP16-13.pdf.

Lowerre-Barbieri, Susan K., et al. "A Comparison of a Validated Otolith Method to Age Weakfish, Cynoscion Regalis, with the Traditional Scale Method." ODU Digital Commons, 1994, https://digitalcommons.odu.edu/oeas fac pubs/38/?utm source=digitalcommons.odu.edu $\% 2$ Foeas fac pubs $\% 2$ F38 \&utm medium=PDF\&utm campaign=PDFCoverPages. 
Maceina, M. J., and S. M. Sammons. "An Evaluation of Different Structures to Age Freshwater Fish from a Northeastern Us River.” Wiley Online Library, John Wiley \& Sons, Ltd, 27 July 2006, https://onlinelibrary.wiley.com/doi/10.1111/j.1365-2400.2006.00497.x.

Maraldo, David C., and Hugh R. MacCrimmon. "Comparison of Ageing Methods and Growth Rates for Largemouth Bass, Micropterus Salmoides Lacépède, from Northern Latitudes.” Environmental Biology of Fishes, Kluwer Academic Publishers, Aug. 1979, https://link.springer.com/article/10.1007/BF00005483.

MarAlliance. “"How Old Are You?" Is Not a Straightforward Question for Sharks.” MarAlliance, 9 Jan. 2018, https://maralliance.org/2018/01/09/how-old-are-you-is-not-a-straightforward-question-for-sharks/.

McIntyre, Peter B., et al. "Fish Extinctions Alter Nutrient Recycling in Tropical Freshwaters." PNAS, National Academy of Sciences, 13 Mar. 2007, https://www.pnas.org/content/104/11/4461.short.

Murie, Debra J., et al. "Evaluation of Finrays as a Non-Lethal Ageing Method for Protected Goliath Grouper Epinephelus Itajara.” Endangered Species Research, 1 July 2009, http://www.int-res.com/abstracts/esr/v7/n3/p213$\underline{220 / .}$.

NOAA. "Aquatic Food Webs.” Aquatic Food Webs | National Oceanic and Atmospheric Administration, 1 Feb. 2019, https://www.noaa.gov/education/resource-collections/marine-life/aquatic-food-webs.

NOAA Fisheries. "Age and Growth.” NOAA, 2020, https:/www.fisheries.noaa.gov/national/science-data/age-andgrowth.

NOAA Fisheries. "Fisheries Economics of the United States, 2016 Fact Sheet." NOAA, 2 June 2020, https://www.fisheries.noaa.gov/resource/document/fisheries-economics-united-states-2016-fact-sheet.

NOAA Fisheries. "Understanding Fisheries Management in the United States.” NOAA, https://www.fisheries.noaa.gov/insight/understanding-fisheries-management-united-states.

Pouilly, Marc, et al. "Geographical Origin of Amazonian Freshwater Fishes Fingerprinted by 87Sr/86Sr Ratios on Fish Otoliths and Scales.” ACS Publications, 27 June 2014, https://pubs.acs.org/doi/full/10.1021/es500071w.

Ramel, Gordon. "Fish Scales 101: Placoid, Ganoid \& Other Types of Scale Explained.” Earth Life, 24 July 2020 , https://www.earthlife.net/fish/scales.html.

Rawat, Prashant, et al. "Structural and Mechanical Properties of Fish Scales for the Bio-Inspired Design of Flexible Body Armors: A Review." Acta Biomaterialia, Elsevier, Feb. 2021, https://www.sciencedirect.com/science/article/abs/pii/S174270612030708X?dgcid=rss sd all\#: :text=Fish\%20scal es $\% 20$ can $\% 20$ be $\% 20$ four,iv $\% 20$ placoid $\% 20 \% 5$ B $5 \% 5$ D.

Rijnsdorp, Adriaan D., et al. "Resolving the Effect of Climate Change on Fish Populations." OUP Academic, Oxford University Press, 2 Apr. 2009, https://doi.org/10.1093/icesjms/fsp056.

“Snap-a-Striper." Gulf Of Maine Research Institute, https://gmri.org/projects/snap-striper/. 
Takagi, Y., et al. "Scale Regeneration of Tilapia (Oreochromis Niloticus) under Various Ambient and Dietary Calcium Concentrations." AGRIS, 1 Jan. 1989, https://agris.fao.org/agris-search/search.do?recordID=GB8906105.

Teh, Lydia C L, and U R Sumaila. "Contribution of Marine Fisheries to Worldwide Employment.” Wiley Online Library, John Wiley \& Sons, Ltd, 7 Dec. 2011, https://onlinelibrary.wiley.com/doi/abs/10.1111/j.14672979.2011.00450.x.

Trueman, Clive N., and Andy Moore. "Use of the Stable Isotope Composition of Fish Scales for Monitoring Aquatic Ecosystems." Terrestrial Ecology, Elsevier, 11 Oct. 2007, https://www.sciencedirect.com/science/article/abs/pii/S193679610701010X?via\%3Dihub.

Volff, J-N. "Genome Evolution and Biodiversity in Teleost Fish." Nature News, Nature Publishing Group, 22 Dec. 2004, https://www.nature.com/articles/6800635.

Welch, Timothy J., et al. "Precision and Relative Accuracy of Striped Bass Age Estimates from Otoliths, Scales, and Anal Fin Rays and Spines.” American Fisheries Society, John Wiley \& Sons, Ltd, Aug. 1993, https://afspubs.onlinelibrary.wiley.com/doi/abs/10.1577/1548$\underline{8675 \% 281993 \% 29013 \% 3 \mathrm{C} 0616 \% 3 \mathrm{APARAOS} \% 3 \mathrm{E} 2.3 . \mathrm{CO} \% 3 \mathrm{~B} 2 .}$ 